\title{
Probing bino-wino coannihilation dark matter below the neutrino floor at the $\mathrm{LHC}$
}

\author{
Guang Hua Duan, ${ }^{1,2,3}$ Ken-ichi Hikasa, ${ }^{4}$ Jie Ren, ${ }^{2,3, \dagger}$ Lei Wu, ${ }^{1, *}$ and Jin Min Yang ${ }^{2,3,4}$ \\ ${ }^{1}$ Department of Physics and Institute of Theoretical Physics, Nanjing Normal University, \\ Nanjing, Jiangsu 210023, China \\ ${ }^{2}$ CAS Key Laboratory of Theoretical Physics, Institute of Theoretical Physics, \\ Chinese Academy of Sciences, Beijing 100190, China \\ ${ }^{3}$ School of Physical Sciences, University of Chinese Academy of Sciences, Beijing 100049, China \\ ${ }^{4}$ Department of Physics, Tohoku University, Sendai 980-8578, Japan
}

(Received 19 April 2018; published 9 July 2018)

\begin{abstract}
In the minimal supersymmetric standard model (MSSM), the bino-wino coannihilation provides a feasible way to accommodate the observed cosmological dark matter (DM) relic density. However, such a scenario usually predicts a very small DM-nucleon scattering cross section that is below the neutrino floor, and can not be tested by DM direct detection experiments. In this work, we investigate the discovery potential of this bino-wino co-annihilation region by searching for the soft dilepton events from the process $p p \rightarrow \chi_{2}^{0}\left(\rightarrow \ell^{+} \ell^{-} \chi_{1}^{0}\right) \chi_{1}^{ \pm}+$jets at the LHC. We find that the mass of the winolike $\chi_{2}^{0}$ can be probed up to about 310 (230) $\mathrm{GeV}$ at $2 \sigma(5 \sigma)$ level for an integrated luminosity $\mathcal{L}=300 \mathrm{fb}^{-1}$. In the future HL-LHC with $3000 \mathrm{fb}^{-1}$ luminosity, the corresponding mass limits can be pushed up to 430 (330) $\mathrm{GeV}$.
\end{abstract}

DOI: 10.1103/PhysRevD.98.015010

\section{INTRODUCTION}

Dark matter accounts for about $27 \%$ of the global energy budget in the Universe. However, it has not yet been directly detected and its identity remains a mystery. The paradigm of weakly interacting massive particles (WIMPs) is one of the most compelling versions and motivates various searches for WIMP DM in the underground, collider, and satellite experiments [1].

Recently, the direct detections of nucleon-WIMP DM scattering have reached impressive sensitivities. Their null results excluded the spin-independent cross section above $7.7 \times 10^{-47} \mathrm{~cm}^{2}$ for a DM mass of $35 \mathrm{GeV}$ [2] and the spin-dependent cross section above $4.1 \times 10^{-41} \mathrm{~cm}^{2}$ for a DM mass of $40 \mathrm{GeV}$ [3], which are approaching the neutrino floor. Below this irreducible neutrino background, the coherent neutrino scattering will mimic the DM signal. On the other hand, the WIMP DM may interact with the nucleons at loop levels [4] or annihilate with a light species

\footnotetext{
*Corresponding author. leiwu@njnu.edu.cn

Corresponding author. renjie@itp.ac.cn

Published by the American Physical Society under the terms of the Creative Commons Attribution 4.0 International license. Further distribution of this work must maintain attribution to the author(s) and the published article's title, journal citation, and DOI. Funded by SCOAP ${ }^{3}$.
}

that is nearby in mass [5]. Then, the correlation between the DM annihilation cross section for relic density and the DMnucleon scattering cross section for direct detection will become weak or even disappear. Such a kind of WIMP DM can, therefore, successfully meet the requirement of the observed relic density and escape the current direct detections.

The minimal supersymmetric standard model (MSSM) is one of the most promising extensions of the SM, in which the lightest neutralino $\left(\chi_{1}^{0}\right)$ can be a natural WIMP dark matter if $R$-parity is conserved. Provided that $\chi_{1}^{0}$ is binolike,. ${ }^{1}$ there are usually three ways to achieve the correct DM relic density:

(i) $\chi_{1}^{0}$ may be a well-tempered neutralino [6], i.e., an appropriate mixture of bino and Higgsino (or bino, Higgsino, and wino). However, a rather large portion of the well-tempered bino-Higgsino scenario is in tension with current XENON1T and LUX data [7-9], and the entire parameter space could be covered by future direct detections, such as LUXZEPLIN [10]. Even for the "blind spots" [11], they may be unblinded with the collective efforts in future DM searches [12];

\footnotetext{
${ }^{1}$ The lightest neutralino $\chi_{1}^{0}$ is a linear combination of the gauge-eigenstates $\left(\tilde{B}, \tilde{W}, \tilde{H}_{d}^{0}, \tilde{H}_{u}^{0}\right): \chi_{1}^{0}=N_{11} \tilde{B}+N_{12} \tilde{W}+N_{13} \tilde{H}_{d}^{0}+$ $N_{14} \tilde{H}_{u}^{0}$, where $N$ is a unitary $4 \times 4$ matrices that diagonalizes the neutralino mass matrix in Eq. (1). When $N_{11}^{2}>$ $\max \left\{N_{12}^{2}, N_{13}^{2}+N_{14}^{2}\right\}, \chi_{1}^{0}$ is called binolike.
} 
(ii) $\chi_{1}^{0}$ can annihilate resonantly via $Z$-funnel, Higgsfunnel or $H / A$-funnel, if its mass is close to half of the mass of the funnel-particle. These funnel regions will be likely covered by future (in)direct detections;

(iii) $\chi_{1}^{0}$ can coannihilate with other light sparticles (e.g., a stop [13], stau [14], wino [15], or gluino [16]). This requires their masses to be nearly degenerate. Due to the sterility of binolike DM, the DM-nucleon scattering rates in these coannihilation scenarios are usually below the neutrino floor.

Given the strong LHC constraints on the colored SUSY particles and the nonobservation of DM in direct detections, the bino-wino coannihilation is one of the feasible ways that can provide the correct DM relic density and escape the direct detections $[15,17,18]$. It may happen in the so-called split supersymmetry [19-22], the spread supersymmetry [23] or supersymmetric GUT models with nonuniversal gaugino masses at the boundary [15]. Besides, such a scenario is also favored by the very recent likelihood analysis of the pMSSM11 under various experimental constraints [24].

In this work, we will investigate the potential of probing such a bino-wino coannihilation scenario at the LHC. The ATLAS and CMS collaborations have widely searched for the electroweakinos in different final states $[25,26]$. However, the sensitivity of these channels depends on the mass difference $(\Delta m)$ between NLSP and LSP. When $\Delta m$ becomes small, the productions of electroweakinos would lead to a small missing energy and soft leptons in the final states, even a displaced vertex $[27,28]$ at the LHC. In order to detect those compressed electroweakinos with the mass splitting $\Delta m<5 \mathrm{GeV}$, one often uses a visible object $X$ $(X=j, \gamma, Z$ ) from initial state radiation (ISR) to boost the electroweakino pair system and enhance $\mathscr{E}_{T}$ in the event, while the other decay products still remain soft [29-34]. For the mass splitting $5 \mathrm{GeV}<\Delta m<50 \mathrm{GeV}$, the low transverse momentum lepton events from the decays of compressed electroweakinos can be used to effectively distinguish the signal from the SM backgrounds [35-39]. We will utilize such soft dilepton events from the process $p p \rightarrow \chi_{2}^{0}\left(\rightarrow \ell^{+} \ell^{-} \chi_{1}^{0}\right) \chi_{1}^{ \pm}+$jets to probe the bino-wino coannihilation at the LHC. Besides, there is no upper limit on the number of jets in our analysis. Although a veto on the second and other jets with $p_{T}>20 \mathrm{GeV}$ in the monojet analysis can sufficiently suppress the $t \bar{t}$ background, we will lose too many signal events [40]. Instead, one can apply a cut on the transverse mass $m_{T}\left(\ell_{i}, \mathbb{E}_{T}\right)$ to be below $70 \mathrm{GeV}$, which can also remove the $t \bar{t}$ background events efficiently but do not hurt the signal too much. Then, being inclusive in jets can increase the signal events and give us better sensitivity than the conventional monojet method.

The structure of this paper is organized as follows. In Sec. II, we confront the bino-wino coannihilation scenario with the direct detections. In Sec. III, we present the discovery potential of this scenario by analyzing the soft dilepton events at the LHC. Finally, we draw our conclusions in Sec. IV.

\section{BINO-WINO COANNIHILATION AND DIRECT DETECTIONS}

In the MSSM, the mass matrix for neutralinos $\chi_{1,2,3,4}^{0}$ in gauge-eigenstate basis $\left(\tilde{B}, \tilde{W}, \tilde{H}_{d}^{0}, \tilde{H}_{u}^{0}\right)$ is given by

$$
M_{\chi^{0}}=\left(\begin{array}{cccc}
M_{1} & 0 & -c_{\beta} s_{W} m_{Z} & s_{\beta} s_{W} m_{Z} \\
0 & M_{2} & c_{\beta} c_{W} m_{Z} & s_{\beta} c_{W} m_{Z} \\
-c_{\beta} s_{W} m_{Z} & c_{\beta} c_{W} m_{Z} & 0 & -\mu \\
s_{\beta} s_{W} m_{Z} & -s_{\beta} c_{W} m_{Z} & -\mu & 0
\end{array}\right)
$$

where $s_{\beta}=\sin \beta$ and $c_{\beta}=\cos \beta . M_{1,2}$ are soft-breaking mass parameters for bino and wino, and $\mu$ is the Higgsino mass parameter. This mass matrix can be diagonalized by a unitary $4 \times 4$ matrices $N$ [41]. The chargino mass matrix in the gauge-eigenstates basis $\left(\tilde{W}^{+}, \tilde{H}_{u}^{+}, \tilde{W}^{-}, \tilde{H}_{d}^{-}\right)$is given by

$$
\mathrm{M}_{\chi^{ \pm}}=\left(\begin{array}{cc}
0 & X^{T} \\
X & 0
\end{array}\right)
$$

with

$$
X=\left(\begin{array}{cc}
M_{2} & \sqrt{2} s_{\beta} m_{W} \\
\sqrt{2} c_{\beta} m_{W} & \mu
\end{array}\right)
$$

Here the mass matrix $X$ can be diagonalized by two unitary $2 \times 2$ matrices $U$ and $V$ [41].

In the limit of $\left|M_{1}\right| \simeq\left|M_{2}\right| \ll \mu$, one can have the masses of $\chi_{1,2}^{0}$ and $\chi_{1}^{ \pm}$at $\mathcal{O}(1 / \mu)$ :

$$
\begin{gathered}
m_{\chi_{1}^{0}} \simeq\left|M_{1}\right|-\frac{m_{W}^{2} \tan ^{2} \theta_{W}}{\mu} \sin 2 \beta, \\
m_{\chi_{2}^{0}, \chi_{1}^{ \pm}} \simeq\left|M_{2}\right|-\frac{m_{W}^{2}}{\mu} \sin 2 \beta .
\end{gathered}
$$

with the mass splitting

$\Delta m_{\chi_{2}^{0}, \chi_{1}^{ \pm}-\chi_{1}^{0}} \simeq\left(\left|M_{2}\right|-\left|M_{1}\right|\right)-\frac{m_{W}^{2}\left(1-\tan ^{2} \theta_{W}\right)}{\mu} \sin 2 \beta$.

Due to the small couplings of Higgs/ $Z$ boson with the binolike $\chi_{1}^{0}$, the annihilation rate of $\chi_{1}^{0} \chi_{1}^{0}$ into the SM particles is highly suppressed. Then, the coannihilation of $\chi_{1}^{0}$ with the winolike electroweakinos $\chi_{1}^{ \pm}, \chi_{2}^{0}$ will become essential in producing the correct DM relic density. 


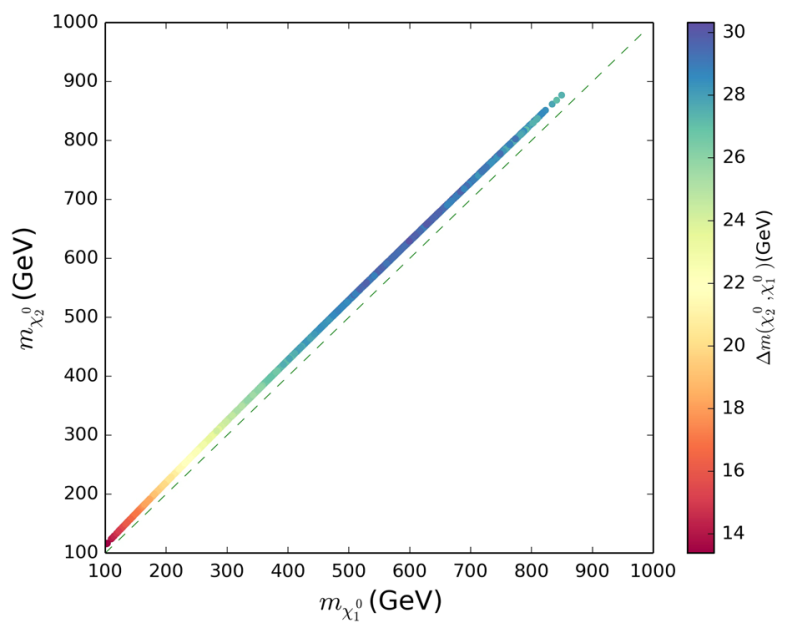

FIG. 1. The scatter plots of bino-wino coannihilation on the plane of $m_{\chi_{1}^{0}}$ versus $m_{\chi_{2}^{0}}$. All samples satisfy the constraints (i) and (ii).

We scan over the relevant parameter space for the binowino coannihilation and examine it under the DM direct detections:

$$
100 \mathrm{GeV}<\left|M_{1,2}\right|<1 \mathrm{TeV}, \quad 1<\tan \beta<60 .
$$

We assume a common mass $\tilde{M}$ for the Higgsino mass parameter $\mu$, gluino mass parameter $M_{3}$, the pseudoscalar Higgs mass $m_{A}$, the first-two-generation squark soft mass parameters $m_{\tilde{q}_{1,2}}$, and all slepton soft mass parameters $m_{\tilde{\ell}_{1,2,3}}$ and fix $\tilde{M}=5 \mathrm{TeV}$ for simplicity. In the MSSM with moderate $\tan \beta$ and large $m_{A}$, the Higgs mass is given by $[42,43]$

$m_{h}^{2} \simeq M_{Z}^{2} \cos ^{2} 2 \beta+\frac{3 m_{t}^{4}}{4 \pi^{2} v^{2}} \ln \frac{M_{S}^{2}}{m_{t}^{2}}+\frac{3 m_{t}^{4}}{4 \pi^{2} v^{2}} \frac{X_{t}^{2}}{M_{S}^{2}}\left(1-\frac{X_{t}^{2}}{12 M_{S}^{2}}\right)$,

where $v=174 \mathrm{GeV}, X_{t} \equiv A_{t}-\mu \cot \beta$, and $M_{S}$ is the average stop mass scale defined by $M_{S}=\sqrt{m_{\tilde{t}_{1}} m_{\tilde{t}_{2}}}$. From Eq. (8), it can be seen that the heavy multi-TeV stops and/or large Higgs-stop trilinear soft-breaking coupling are required to achieve a observed $125 \mathrm{GeV}$ Higgs mass. We take the third-generation squark soft masses $M_{\tilde{Q}_{3 L}}=M_{\tilde{t}_{3 R}}=M_{\tilde{b}_{3 R}}=5 \mathrm{TeV}$ and vary the trilinear parameters in the range of $\left|A_{t, b}\right|<3 \mathrm{TeV}$. We evaluate the mass spectrum and branching ratios of the sparticles with SUSY-HIT [44] and impose the following constraints:

(i) The light $C P$-even Higgs boson mass should be within the range of $125 \pm 3 \mathrm{GeV}$.

(ii) The DM relic density should satisfy the observed value $0.1186 \pm 0.0020$ [45] within $2 \sigma$ range, which is computed by MicrOMEGAs 4.3.2 [46].
In Fig. 1, we show the samples that satisfy the above constraints (1) and (2) on the plane of $m_{\chi_{1}^{0}}$ versus $m_{\chi_{2}^{0}}$. In order for the coannihilation partner $\chi_{1}^{ \pm}$to have a significant effect on the dark matter relic density, its mass should be close to $\chi_{1}^{0}$ with a splitting $\Delta m_{\chi_{1}^{ \pm}-\chi_{1}^{0}} / m_{\chi_{1}^{0}} \simeq 3 \%-10 \%$. The dominant contribution to achieving the relic density comes from $\chi_{2}^{0} \chi_{1}^{ \pm}$coannihilation $(\sim 50 \%)$, which is followed by $\chi_{1}^{+} \chi_{1}^{-} \quad(\sim 30 \%)$ and $\chi_{1}^{0} \chi_{1}^{ \pm} / \chi_{2}^{0} / \chi_{1}^{0}(\sim 20 \%)$ coannihilation processes. When $\Delta m_{\chi_{1}^{ \pm}-\chi_{1}^{0}} \ll M_{W}$, the winolike chargino decays into a pair of fermions and a binolike neutralino, via an off-shell $W$. The decay width of this process is given by,

$$
\Gamma\left(\chi_{1}^{ \pm} \rightarrow f \bar{f}^{\prime} \chi_{1}^{0}\right) \simeq \frac{4 \alpha^{2}}{15 \pi} \frac{\left(\Delta m_{\chi_{1}^{ \pm}-\chi_{1}^{0}}\right)^{3}}{\mu^{2}} \frac{\sin ^{2} 2 \beta}{\sin ^{2} 2 \theta_{W}} .
$$

Very recently, ATLAS has published its updated analysis of searching for disappearing track events from the compressed chargino decay [47], which excludes the chargino with lifetime longer than about $0.2 \mathrm{~ns}$ and masses less than $430 \mathrm{GeV}$. We checked that our samples can avoid such ATLAS limits because the lifetimes of our charginos are much shorter than $0.01 \mathrm{~ns}$.

Next, we calculate the spin-independent (SI) and spindependent (SD) neutralino LSP-nucleon scattering cross sections, in which the form factors of proton and neutron are taken as,

$$
\begin{aligned}
& f_{u}^{p} \approx 0.020, \quad f_{d}^{p} \approx 0.026, \quad f_{s}^{p} \approx 0.13 \\
& f_{u}^{n} \approx 0.014, \quad f_{d}^{n} \approx 0.036, \quad f_{s}^{n} \approx 0.13
\end{aligned}
$$

In Fig. 2, we show the direct detection results for all samples satisfying the constraints (1) and (2). We can see that the SI cross sections for bino-wino coannihilation samples are very small because of the tiny couplings of bino-like LSP with the Higgs boson ${ }^{2}$ Most of them are even below the neutrino floor so that they can escape the existing strong limits from PandaX-II(2017), XENON1T(2017), XENON100(2016), LUX(2017) and the future direct detection experiments, such as LUX-ZEPLIN, which will improve the current sensitivity of LUX by about two orders of magnitude. Besides, it should be noted that the SD cross section of DM scattering off a neutron $\sigma_{\chi, n}^{\mathrm{SD}}$ is largely determined by $Z$-boson exchange and is sensitive to the Higgsino asymmetry, which can be approximately given by

$$
\sigma_{\chi, n}^{\mathrm{SD}} \simeq 3.1 \times 10^{-4} \mathrm{pb} \cdot\left(\frac{\left|N_{13}\right|^{2}-\left|N_{14}\right|^{2}}{0.1}\right)^{2} .
$$

\footnotetext{
${ }^{2}$ In the top panel of Fig. 2, the separate bands for SI scattering cross section are caused by our scan method, in which we scan the parameter $\operatorname{space}$ for $\operatorname{sign}\left(M_{1} / M_{2}\right)= \pm 1$ independently. The gap between two regions will become narrow and disappear when the number of samples for both $\operatorname{sign}\left(M_{1} / M_{2}\right)= \pm 1$ increase enough.
} 

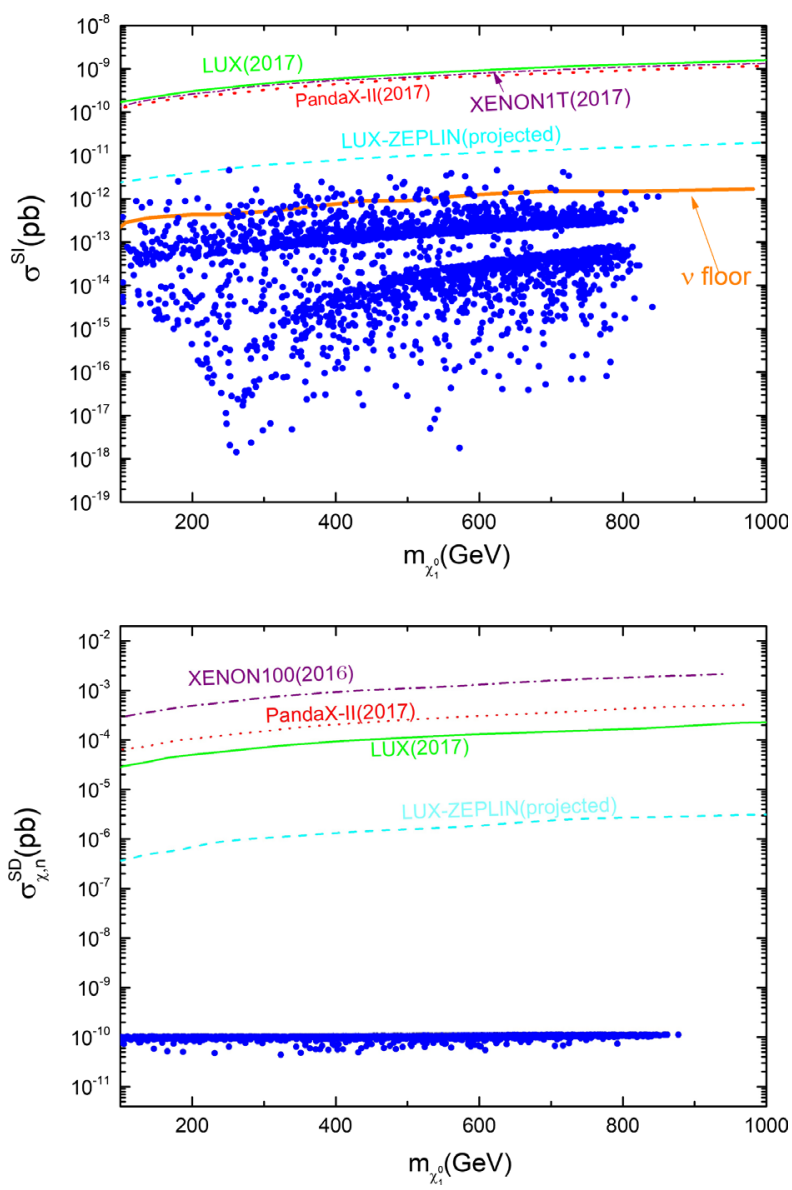

FIG. 2. Same as Fig. 1, but for the spin-independent and spindependent neutralino LSP-nucleon scattering cross sections. The observed $90 \%$ C.L. upper limits are from PandaX-II with a total exposure of $90.4 \mathrm{~kg} \times \mathrm{yr}$ [3], XENON1T (2017) with a total exposure of $97.1 \mathrm{~kg} \times \mathrm{yr}$ [2], XENON100 (2016) with a total exposure of $48 \mathrm{~kg} \times \mathrm{yr}$ [48] and LUX (2017) with a total exposure of $129.5 \mathrm{~kg} \times \mathrm{yr}$ [49]. The projected LUX-ZEPLIN with a exposure of $153.4 \times 10^{2} \mathrm{~kg} \times \mathrm{yr}$ [50] sensitivity limits are plotted.

The values of $\left|N_{13}\right|$ and $\left|N_{14}\right|$ for samples in our scan ranges are usually about $\mathcal{O}\left(10^{-2}\right)$ and $\mathcal{O}\left(10^{-3}\right)$ so that $\sigma_{\chi, n}^{\mathrm{SD}}$ is constantly around $\mathcal{O}\left(10^{-10}\right) \mathrm{pb}$. Thus, it is meaningful to explore the observability of this bino-wino coannihilation in other experiments, such as the LHC.

\section{SEARCH FOR SOFT DILEPTON EVENTS AT THE LHC}

We calculate the cross section of the process $p p \rightarrow \chi_{2}^{0} \chi_{1}^{ \pm}$ for $\tan \beta=30$ at $13 \mathrm{TeV}$ LHC and show the result in Fig. 3. We can see that the cross section can reach about $10 \mathrm{pb}$ at $M_{2}=100 \mathrm{GeV}$. When $M_{2}$ becomes large, the cross section decreases rapidly.

Now we perform a detailed Monte Carlo simulation of the process $p p \rightarrow \chi_{2}^{0} \chi_{1}^{ \pm}+$jets with the sequent decay channel

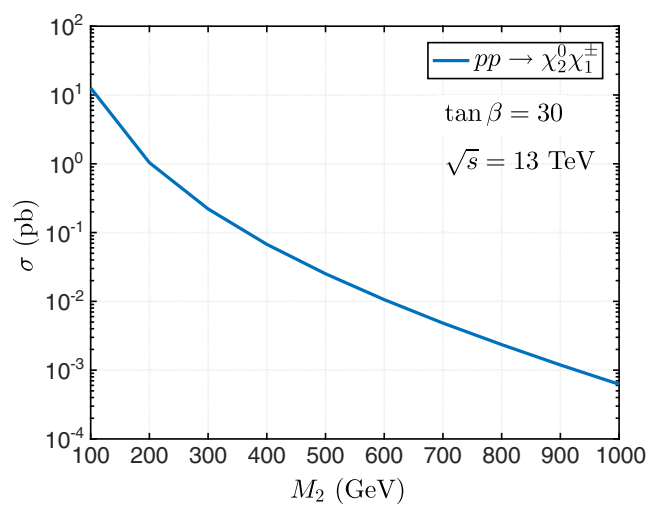

FIG. 3. The cross section of $p p \rightarrow \chi_{2}^{0} \chi_{1}^{ \pm}$at the $13 \mathrm{TeV}$ LHC. Here $M_{1}=M_{2}-30 \mathrm{GeV}$ and $\tan \beta=30$.

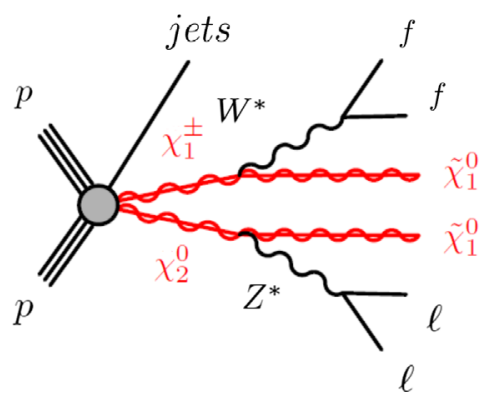

FIG. 4. Diagram representing for the process $p p \rightarrow \chi_{2}^{0} \chi_{1}^{ \pm}+$jets, $\chi_{2}^{0} \rightarrow Z^{*} \chi_{1}^{0} \rightarrow \ell^{+} \ell^{-} \chi_{1}^{0}$.

$\chi_{2}^{0} \rightarrow Z^{*} \chi_{1}^{0} \rightarrow \ell^{+} \ell^{-} \chi_{1}^{0}$ at the LHC, as shown in Fig. 4. Contrary to the monojet analysis for the compressed electroweakinos, there is no upper limit on the number of jets in our analysis. The main SM backgrounds for our signal are events from Drell-Yan (DY) processes with subsequent decays $\gamma / Z^{*} \rightarrow \tau^{+} \tau^{-} \rightarrow \ell^{+} \ell^{-} \nu_{\ell} \bar{\nu}_{\ell} \nu_{\tau} \bar{\nu}_{\tau}$, and fully leptonic $t \bar{t}$ decays. Smaller backgrounds are diboson $(V V)$ processes like $W^{+} W^{-}$and single top production like $t W$. In additional, the nonprompt leptons can also mimic our signal, which mainly arise from $W+j$ events. In Ref. [51], several parametrizations of the lepton fake rates are extracted from comparison with the CMS data. At low $p_{T}$, where the bulk of our soft leptons distribute, the fake rate is quoted as $\mathcal{O}\left((0.6--3) \times 10^{-5}\right)$. So, the background from fake leptons is $\mathcal{O}(1 \%)$ of the dominant backgrounds. On the other hand, a low invariant mass cut $4 \mathrm{GeV}<m_{\ell \ell}<50 \mathrm{GeV}$ will further reduce such nonprompt backgrounds as the distribution from $W+j$ events with a fake lepton has a higher value of $m_{\ell \ell}$ than the signal [35]. In our simulations, we focus on the prompt SM backgrounds.

We generate the parton level events of signal and backgrounds with MADGRAPH5_aMC@NLO [52] and implement the shower and hadronization by using PYTHIA-8.2 [53]. The detector effects are simulated by DeLPHEs [54]. The jet clustering is performed by FASTJET [55] with the anti- $k_{t}$ algorithm [56]. Due to the limitation of CPU, the 
TABLE I. The cut flow for the cross sections of the signal and backgrounds at the $13 \mathrm{TeV}$ LHC before using the signal regions. The benchmark point is $m_{\chi_{1}^{0}}=137.1 \mathrm{GeV}, m_{\chi_{2}^{0}}=m_{\chi_{1}^{ \pm}}=153.3 \mathrm{GeV}, \tan \beta=34$. The cross sections are in unit of fb.

\begin{tabular}{|c|c|c|c|c|}
\hline Cuts & $t \bar{t}$ & $\begin{array}{c}\text { Backgrounds } \\
\text { Drell-Yan }\end{array}$ & Diboson & $\begin{array}{c}\text { Signal } \\
\chi_{2}^{0} \chi_{1}^{ \pm} \\
\end{array}$ \\
\hline $\mathbb{E}_{T}>125 \mathrm{GeV}$ & 45108.12 & 1636.39 & 2664.94 & 662.84 \\
\hline$N(j)>0, N(b)=0$ & 8776.16 & 1309.35 & 1903.41 & 528.24 \\
\hline$N(\ell)=2, \mathrm{OSSF}, p_{T}\left(\ell_{1,2}\right) \in[5,30] \mathrm{GeV}$ & 57.45 & 31.45 & 5.77 & 5.45 \\
\hline$m_{\ell \ell} \in[4,50] \mathrm{GeV}, m_{\ell \ell} \notin[9,10.5] \mathrm{GeV}$ & 44.62 & 29.34 & 4.43 & 4.35 \\
\hline$H_{T}>100 \mathrm{GeV}, \mathbb{E}_{T} / H_{T} \in[0.6,1.4]$ & 28.14 & 23.47 & 3.32 & 3.59 \\
\hline$M_{T}\left(l, \mathbb{E}_{T}\right)<70 \mathrm{GeV}$ & 8.83 & 21.57 & 0.98 & 2.77 \\
\hline$M_{\tau \tau} \notin[0,160] \mathrm{GeV}$ & 6.12 & 7.21 & 0.75 & 2.123 \\
\hline
\end{tabular}

signal, Drell-Yan and diboson production processes are matched up to one jet by using the MLM-scheme with merging scale $Q=30 \mathrm{GeV}$. We normalize the cross sections of the processes $p p \rightarrow \chi_{1}^{ \pm} \chi_{2}^{0}$ and $p p \rightarrow t \bar{t}$ to their NLO order [57] and the NNLO + NNLL order values [58], respectively. The event selections are performed within the framework of CHECKMATE2 [59].

In our object reconstruction, we require the leptons to satisfy $p_{\mathrm{T}} \in[5,30] \mathrm{GeV}$ and $|\eta|<2.5$. The leptons are isolated within a cone in $\eta-\phi$ space of radius $\Delta R=$ $\sqrt{\Delta \eta^{2}+\Delta \phi^{2}}<0.3$ if the sum of the transverse momenta within the cone is less than $5 \mathrm{GeV}$ and the ratio of it to the $p_{\mathrm{T}}$ of the lepton is less than 0.5. Jets are clustered from calorimeter towers using anti- $k_{\mathrm{T}}$ jet clustering algorithm with a distance parameter $R=0.4$. Jets are required to have $p_{\mathrm{T}}>25 \mathrm{GeV}$ and $|\eta|<2.4$. We assume the $b$-jet tagging efficiency as $80 \%$ and include a misidentification $10 \%$ for light jets.

The following cuts are applied to differentiate signal from backgrounds:

(i) A large missing transverse energy $\mathbb{E}_{T}>125 \mathrm{GeV}$ is required.

(ii) At least one jet but a veto on events with $p_{T}(b)>$ $25 \mathrm{GeV}$ is imposed to reduce the $t \bar{t}$ background.

(iii) A pair of opposite-sign same-flavor (OSSF) leptons are required. Two leptons should have a small transverse momentum $5 \mathrm{GeV}<p_{T}\left(\ell_{1,2}\right)<30 \mathrm{GeV}$.

(iv) The invariant mass of the two leptons is required in the range $4 \mathrm{GeV}<m_{\ell \ell}<50 \mathrm{GeV}$ but $m_{\ell \ell} \notin[9,10.5] \mathrm{GeV}$, which can reduce the diboson background and the potential soft lepton events from Drell-Yan and $J / \psi$ and $\Upsilon$ decays.

(v) The transverse hadronic energy $H_{T}>100 \mathrm{GeV}$, which is defined as the scalar sum of the transverse momenta of the selected jets.

(vi) The QCD multijet background can be efficiently suppressed by the requirement of large $\mathbb{E}_{T}$ and two leptons. Besides, we apply a cut $0.6<\mathbb{E}_{T} / H_{T}<1.4$ to reject the residual QCD multijet events, which mostly sit at low $\mathbb{E}_{T} / H_{T}$ values [60]. This is because that large $\mathbb{E}_{T}$ from the mismeasurement of QCD jests needs the mismeasured jets to have a large momentum as well, and therefore the $\mathscr{E}_{T} / H_{T}$ variable is quite small in QCD. In this respect, the lower cut on $\mathbb{E}_{T} / H_{T}$ is necessary, but not sufficient to suppress QCD. Together with the requirement of $\mathbb{E}_{T}$ and two leptons, the QCD multijet background becomes negligible.

(vii) The transverse mass $m_{T}\left(\ell_{i}, \mathbb{E}_{T}\right)<70 \mathrm{GeV}$ is used to further suppress $t \bar{t}$ backgrounds.

(viii) The invariant mass $m_{\tau \tau} \notin[0,160] \mathrm{GeV}$ [35]. This observable is used to reject the large background from $\gamma^{*} / Z \rightarrow \tau^{+} \tau^{-}$events with the $\tau$ leptons decay leptonically. Since the $\tau$ leptons from $\gamma^{*} / Z$ boson decays are highly energetic, the direction of the final lepton is approximately the same as that of the parent $\tau$ lepton, which leads to $\vec{p}_{\nu_{i}}=\xi_{i} \vec{p}_{\ell_{i}}$. If the missing energy is due to four neutrinos, one can solve $\xi_{i}$ by using the measured $\mathscr{E}_{T}$ and then reconstruct the $\tau$ lepton momentum through $p_{\tau_{i}}=p_{\nu_{i}}+p_{\ell_{i}}$. It should be noted that the $\tau$ lepton momentum magnitude can take a negative value when the flight direction is opposite to the lepton one. In those events, we set $m_{\tau \tau}$ to be negative. The $\gamma^{*} / Z \rightarrow \tau^{+} \tau^{-}$backgrounds will have a narrow peak around $m_{Z}$ in $m_{\tau \tau}$ distribution, while the signal will be featureless.

After the above selections, we separate the signal events into three regions: $\mathbb{E}_{T} \in[125,200] \mathrm{GeV}, \mathbb{E}_{T} \in[200,250] \mathrm{GeV}$, $\mathbb{E}_{T}>250 \mathrm{GeV}$. In each $\mathbb{E}_{T}$ bin, we further define four signal regions $m_{\ell \ell}=[4,10],[10,20],[20,30],[30,50] \mathrm{GeV}$ to enhance the sensitivity. In Table I, we give the cut flow for the cross sections of the signal and backgrounds at the $13 \mathrm{TeV}$ LHC before using the signal regions. As discussed above, we can see that the Drell-Yan process is the largest background, which is followed by dileptonic $t \bar{t}$ process. The cut on the transverse mass $m_{T}\left(\ell_{i}, \mathbb{E}_{T}\right)<70 \mathrm{GeV}$ can reduce the $t \bar{t}$ background heavily but do not hurt the signal too much. The $m_{\tau \tau}$ cut plays an important role in suppressing the Drell-Yan background.

To estimate the observability, we evaluate the statistical significance $(\alpha)$ by using Poisson formula

$$
\alpha=\sqrt{2 \mathcal{L}\left[\left(\sigma_{S}+\sigma_{B}\right) \ln \left(1+\frac{\sigma_{S}}{\sigma_{B}}\right)-\sigma_{S}\right]} .
$$



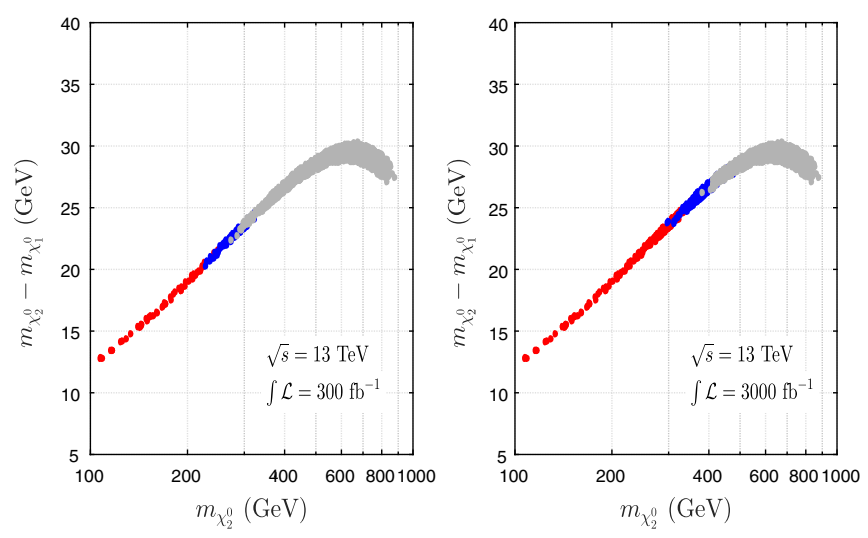

FIG. 5. Same as Fig. 1, but show the statistical significance $(\alpha)$ of the process $p p \rightarrow \chi_{2}^{0} \chi_{1}^{ \pm}+$jets on the plane of $m_{\chi_{2}^{0}}$ versus $m_{\chi_{2}^{0}}-m_{\chi_{1}^{0}}$ at the $13 \mathrm{TeV}$ LHC with the luminosity $\mathcal{L}=300$, $3000 \mathrm{fb}^{-1}$. The gray, blue and red bullets denote $\alpha<2 \sigma, 2 \sigma<$ $\alpha<5 \sigma$ and $\alpha>5 \sigma$, respectively.

where $\sigma_{S}$ and $\sigma_{B}$ are the signal and background cross sections and $\mathcal{L}$ is the integrated luminosity.

In Fig. 5, we present the statistical significance the process $p p \rightarrow \chi_{2}^{0} \chi_{1}^{ \pm}+$jets for the samples allowed by constraints (i)-(ii) on the plane of $m_{\chi_{2}^{0}}$ versus $m_{\chi_{2}^{0}}-m_{\chi_{1}^{0}}$ at the $13 \mathrm{TeV}$ LHC. It can be seen that the significance decreases rapidly when the winolike $\chi_{2}^{0}$ becomes heavy. The mass of $\chi_{2}^{0}$ can be probed up to about $310(230) \mathrm{GeV}$ at $2 \sigma(5 \sigma)$ level for an integrated luminosity $\mathcal{L}=300 \mathrm{fb}^{-1}$. In the future HL-LHC with $3000 \mathrm{fb}^{-1}$ luminosity, the corresponding mass limits can be pushed up to $m_{\chi_{2}^{0}}<430(330) \mathrm{GeV}$.

\section{CONCLUSION}

In the MSSM, the bino-wino coannihilation is one of the important ways to achieve the correct DM relic density. Due to the small couplings of the binolike DM with $Z / h$ bosons, such a scenario can not be probed by the DM direct detections. In this work, we studied the discovery potential of the bino-wino coannihilation scenario by searching for the soft dilepton from the three-body decay of the winolike neutralino $\chi_{2}^{0}$ in the process $p p \rightarrow \chi_{2}^{0} \chi_{1}^{ \pm}+$jets at the LHC. It turns out that the winolike $\chi_{2}^{0}$ can be probed for a mass up to about $310(230) \mathrm{GeV}$ at $2 \sigma(5 \sigma)$ level for an integrated luminosity $\mathcal{L}=300 \mathrm{fb}^{-1}$. The future HL-LHC with $3000 \mathrm{fb}^{-1}$ luminosity will be able to push these mass limits up to 430 (330) $\mathrm{GeV}$.

\section{ACKNOWLEDGMENTS}

We thank Mariana Frank for helpful discussions. This work was supported by the National Natural Science Foundation of China (NNSFC) under Grants No. 11705093 and No. 11675242, by Peng-Huan-Wu Theoretical Physics Innovation Center (11747601), by the CAS Center for Excellence in Particle Physics (CCEPP), by the CAS Key Research Program of Frontier Sciences, and by a Key R\&D Program of Ministry of Science and Technology of China under No. 2017YFA0402200-04.
[1] G. Arcadi, M. Dutra, P. Ghosh, M. Lindner, Y. Mambrini, M. Pierre, S. Profumo, and F. S. Queiroz, Eur. Phys. J. C 78, 203 (2018).

[2] E. Aprile et al. (XENON Collaboration), Phys. Rev. Lett. 119, 181301 (2017).

[3] C. Fu et al. (PandaX-II Collaboration), Phys. Rev. Lett. 118, 071301 (2017).

[4] P. J. Fox and E. Poppitz, Phys. Rev. D 79, 083528 (2009).

[5] K. Griest and D. Seckel, Phys. Rev. D 43, 3191 (1991).

[6] N. Arkani-Hamed, A. Delgado, and G. F. Giudice, Nucl. Phys. B741, 108 (2006).

[7] M. Badziak, M. Olechowski, and P. Szczerbiak, Phys. Lett. B 770, 226 (2017).

[8] M. Abdughani, L. Wu, and J. M. Yang, Eur. Phys. J. C 78, 4 (2018).

[9] H. Baer, V. Barger, and H. Serce, Phys. Rev. D 94, 115019 (2016).

[10] D. S. Akerib et al. (LZ Collaboration), arXiv:1509.02910.

[11] C. Cheung, L. J. Hall, D. Pinner, and J. T. Ruderman, J. High Energy Phys. 05 (2013) 100.

[12] T. Han, F. Kling, S. Su, and Y. Wu, J. High Energy Phys. 02 (2017) 057.
[13] C. Boehm, A. Djouadi, and M. Drees, Phys. Rev. D 62, 035012 (2000).

[14] J. R. Ellis, T. Falk, and K. A. Olive, Phys. Lett. B 444, 367 (1998).

[15] H. Baer, T. Krupovnickas, A. Mustafayev, E. K. Park, S. Profumo, and X. Tata, J. High Energy Phys. 12 (2005) 011.

[16] S. Profumo and C. E. Yaguna, Phys. Rev. D 69, 115009 (2004).

[17] M. Ibe, A. Kamada, and S. Matsumoto, Phys. Rev. D 89, 123506 (2014).

[18] S. Profumo, T. Stefaniak, and L. Stephenson Haskins, Phys. Rev. D 96, 055018 (2017).

[19] N. Arkani-Hamed and S. Dimopoulos, J. High Energy Phys. 06 (2005) 073.

[20] G. F. Giudice and A. Romanino, Nucl. Phys. B699, 65 (2004); B706, 487(E) (2005).

[21] N. Arkani-Hamed, S. Dimopoulos, G. F. Giudice, and A. Romanino, Nucl. Phys. B709, 3 (2005).

[22] J. D. Wells, Phys. Rev. D 71, 015013 (2005).

[23] L. J. Hall and Y. Nomura, J. High Energy Phys. 01 (2012) 082 . 
[24] E. Bagnaschi et al., Eur. Phys. J. C 78, 256 (2018).

[25] M. Aaboud et al. (ATLAS Collaboration), arXiv:1803.02762.

[26] A. M. Sirunyan et al. (CMS Collaboration), arXiv: 1801.03957.

[27] K. Rolbiecki and K. Sakurai, J. High Energy Phys. 11 (2015) 091.

[28] N. Nagata, H. Otono, and S. Shirai, J. High Energy Phys. 10 (2015) 086.

[29] C. Han, A. Kobakhidze, N. Liu, A. Saavedra, L. Wu, and J. M. Yang, J. High Energy Phys. 02 (2014) 049.

[30] P. Schwaller and J. Zurita, J. High Energy Phys. 03 (2014) 060.

[31] H. Baer, A. Mustafayev, and X. Tata, Phys. Rev. D 89, 055007 (2014).

[32] M. Low and L. T. Wang, J. High Energy Phys. 08 (2014) 161.

[33] A. Anandakrishnan, L. M. Carpenter, and S. Raby, Phys. Rev. D 90, 055004 (2014).

[34] D. Barducci, A. Belyaev, A. K. M. Bharucha, W. Porod, and V. Sanz, J. High Energy Phys. 07 (2015) 066.

[35] Z. Han, G. D. Kribs, A. Martin, and A. Menon, Phys. Rev. D 89, 075007 (2014).

[36] J. Bramante, A. Delgado, F. Elahi, A. Martin, and B. Ostdiek, Phys. Rev. D 90, 095008 (2014).

[37] C. Han, L. Wu, J. M. Yang, M. Zhang, and Y. Zhang, Phys. Rev. D 91, 055030 (2015).

[38] H. Baer, A. Mustafayev, and X. Tata, Phys. Rev. D 90, 115007 (2014).

[39] C. Han, D. Kim, S. Munir, and M. Park, J. High Energy Phys. 04 (2015) 132.

[40] M. Drees, M. Hanussek, and J. S. Kim, Phys. Rev. D 86, 035024 (2012).

[41] J. F. Gunion and H. E. Haber, Nucl. Phys. B272, 1 (1986).

[42] M. Carena, J. R. Espinosa, M. Quiros, and C. E. M. Wagner, Phys. Lett. B 355, 209 (1995).

[43] M. Carena, M. Quiros, and C. E. M. Wagner, Nucl. Phys. B461, 407 (1996).

[44] A. Djouadi, M. M. Muhlleitner, and M. Spira, Acta Phys. Pol. B 38, 635 (2007).

[45] P. A. R. Ade et al. (Planck Collaboration), Astron. Astrophys. 594, A13 (2016).
[46] G. Belanger, F. Boudjema, P. Brun, A. Pukhov, S. RosierLees, P. Salati, and A. Semenov, Comput. Phys. Commun. 182, 842 (2011).

[47] M. Aaboud et al. (ATLAS Collaboration), arXiv: 1712.02118 .

[48] E. Aprile et al. (XENON100 Collaboration), Phys. Rev. D 94, 122001 (2016).

[49] D. S. Akerib et al. (LUX Collaboration), Phys. Rev. Lett. 118, 251302 (2017).

[50] D. S. Akerib et al. (LUX-ZEPLIN Collaboration), arXiv: 1802.06039.

[51] D. Curtin, J. Galloway, and J. G. Wacker, Phys. Rev. D 88, 093006 (2013).

[52] J. Alwall, R. Frederix, S. Frixione, V. Hirschi, F. Maltoni, O. Mattelaer, H.-S. Shao, T. Stelzer, P. Torrielli, and M. Zaro, J. High Energy Phys. 07 (2014) 079.

[53] T. Sjöstrand, S. Ask, J. R. Christiansen, R. Corke, N. Desai, P. Ilten, S. Mrenna, S. Prestel, C. O. Rasmussen, and P.Z. Skands, Comput. Phys. Commun. 191, 159 (2015).

[54] J. de Favereau, C. Delaere, P. Demin, A. Giammanco, V. Lemaître, A. Mertens, and M. Selvaggi (DELPHES 3 Collaboration), J. High Energy Phys. 02 (2014) 057.

[55] M. Cacciari, G. P. Salam, and G. Soyez, Eur. Phys. J. C 72, 1896 (2012).

[56] M. Cacciari, G. P. Salam, and G. Soyez, J. High Energy Phys. 04 (2008) 063.

[57] W. Beenakker, M. Klasen, M. Kramer, T. Plehn, M. Spira, and P. M. Zerwas, Phys. Rev. Lett. 83, 3780 (1999); 100, 029901(E) (2008).

[58] M. Czakon and A. Mitov, Comput. Phys. Commun. 185, 2930 (2014).

[59] M. Drees, H. K. Dreiner, J. S. Kim, D. Schmeier, and J. Tattersall, Comput. Phys. Commun. 187, 227 (2015); J. S. Kim, D. Schmeier, J. Tattersall, and K. Rolbiecki, Comput. Phys. Commun. 196, 535 (2015); D. Dercks, N. Desai, J. S. Kim, K. Rolbiecki, J. Tattersall, and T. Weber, Comput. Phys. Commun. 221, 383 (2017).

[60] A. M. Sirunyan et al. (CMS Collaboration), Phys. Lett. B 782, 440 (2018). 